\title{
Clinical Experience of Negative Pressure Wound Therapy with Instillation
}

\author{
Yoichi Nishii \\ Department of Plastic and Reconstructive Surgery, Uji-Tokushukai Medical Center, Uji, Japan
}

Standard negative pressure wound therapy (NPWT) is an established treatment option that is able to promote granulation tissue growth in wounds. However, there is limited evidence for its utility in active or severe infection, which is very common in wounds and tends to require intensive approaches such as serial surgical debridement, antibiosis and medical management. V.A.C. ULTA (KCI, San Antonio, TX, USA), a commercialized medical device for NPWT with instillation, has been introduced in Japan from August 2017. This therapy can control the range of fluid instillation and irrigation (Fig. 1) and has been reported to improve some limitations of standard NPWT. At the 14th Congress of Korea-Japan Plastic and Reconstructive Surgery we showed the clinical results which were obtained in comparatively severe cases by use of ULTA. We also showed that ULTA enabled a shorter period of wound healing compared to standard NPWT.

We retrospectively collected data on 33 patients treated with standard NPWT and 31 patients treated with ULTA for the purpose of wound bed preparation (WBP). The target wounds had wide-ranging causes in various anatomical locations. NPWT treatment had to be discontinued due to obvious infections in seven cases of standard NPWT (21.2\%), compared to only three cases of ULTA (9.7\%).

Appropriate and sufficient debridement is necessary before application of NPWT in all wounds. But in some cases it is difficult to remove necrotic tissue completely. Although NPWT maintains a moderately moist environment by absorption of excess exudate, it also dries tissues with poor blood-flow such as bone or tendon. This could lead to some unwanted side effects in standard NPWT. In cases where WBP is incomplete, reoperations are often necessary. In standard NPWT, such reoperations become easy to happen.

The intermittent instillation function of ULTA is considered to be involved in shortening the time required for wound healing through partial debridement and washing several times per day. If that's the case, more efficient treatment may become possible by adjusting soaking volume and instillation frequency, or adding solutions such as enzymes or disinfectants. The somewhat larger size of ULTA compared to standard NPWT does require patients to stay admitted in the hospital. Furthermore, ULTA is slightly noisy during the instillation period because of motor devices. However, these drawbacks were thought to be minor compared to the advantages of ULTA.

In many cases, ULTA needs to be changed only twice a week just like standard NPWT, and most of the operation is the same. Nevertheless, ULTA was suggested to

\author{
Editorial \\ Received: February 21, 2019 \\ Revised: February 24, 2019 \\ Accepted: February 25, 2019

\section{Corresponding author:} \\ Yoichi Nishii, M.D. \\ Department of Plastic and Reconstructive \\ Surgery, Uji-Tokushukai Medical Center, \\ 145 Ishibashi, Makishima-cho, Uji 611-0041, \\ Japan \\ Tel: +81-774-20-1111 \\ Fax: +81-774-20-2336 \\ E-mail: yoichi241@hotmail.co.jp
}

This is an Open Access article distributed under the terms of the Creative Commons Attribution Non-Commercial License (http://creativecommons.org/licenses/by-nc/4.0/) which permits unrestricted non-commercial use, distribution, and reproduction in any medium, provided the original work is properly cited.

(c) 2019 Korean Wound Management Society 
Nishii Y

The advantages of NPWT with instillation

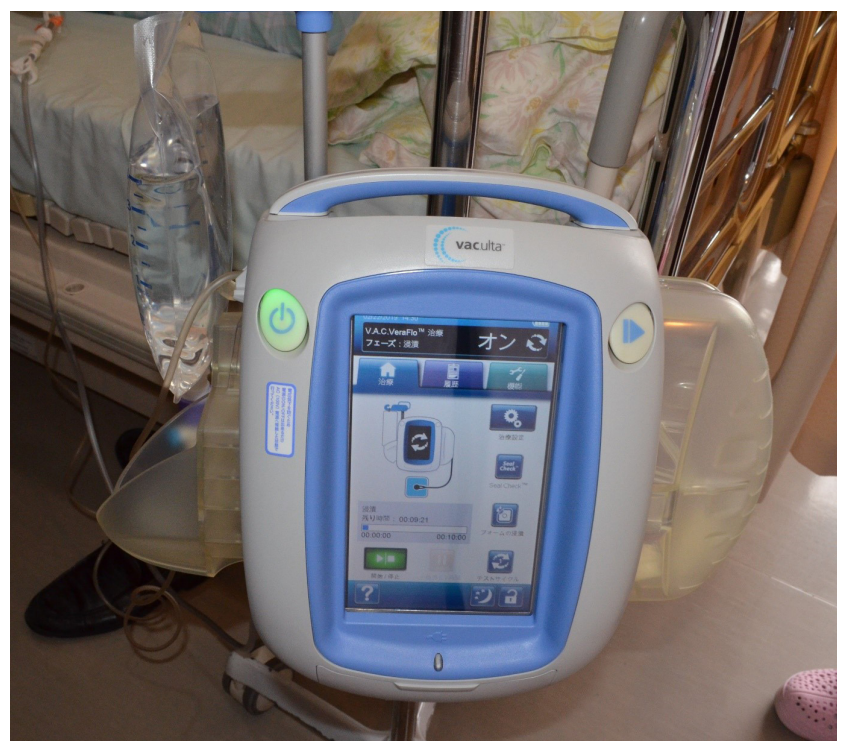

enable complete WBP even in comparatively severe conditions such as infected wounds or exposure of bone or tendon. We consider that NPWT with instillation is a novel and feasible option for WBP in terms of treatment period and improvement of more severe wounds.

\section{Conflict of interest}

No potential conflicts of interest relevant to this article are reported.

\section{Acknowledgments}

Yoichi Nishii https://orcid.org/0000-0003-4193-2077

Fig. 1. Clinical photograph of V.A.C. ULTA device. 